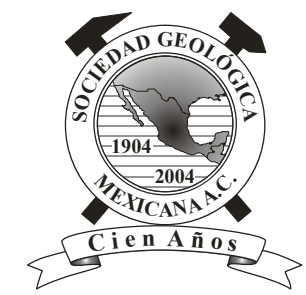

\title{
La relación de subsidencia del terreno InSAR-GPS y el abatimiento del nivel estático en pozos de la zona Metropolitana de la Ciudad de México
}

\author{
Darío Solano-Rojas ${ }^{1}$,Enrique Cabral-Cano ${ }^{2,}$, Antonio Hernández-Espriú ${ }^{3}$, Shimon Wdowinski ${ }^{4}$, \\ Charles DeMets ${ }^{5}$, Luis Salazar-Tlaczani ${ }^{2}$, Giacomo Falorni ${ }^{6}$, Adrian Bohane ${ }^{6}$ \\ ${ }^{1}$ Rosenstiel School of Marine and Atmospheric Science, University of Miami, 4600 Rickenbacker Causeway, Miami, FL 33149, USA. \\ ${ }^{2}$ Departamento de Geomagnetismo y Exploración, Instituto de Geofísica, Universidad Nacional Autónoma de México, Ciudad \\ Universitaria, México D.F., 04510, México. \\ ${ }^{3}$ Grupo de Hidrogeología, División de Ingeniería en Ciencias de la Tierra, Facultad de Ingeniería, Universidad Nacional, Autónoma \\ de México, Ciudad Universitaria, México D.F., 04510, México. \\ ${ }^{4}$ Rosenstiel School of Marine and Atmospheric Science, University of Miami, 4600 Rickenbacker Causeway, Miami, FL 33149, USA. \\ ${ }^{5}$ Department of Geoscience, University of Wisconsin, 1215, Dayton St., Madison, WI 53706, USA. \\ ${ }^{6}$ TRE Canada Inc. 475 W. Georgia Street, Vancouver, BC V6B 4M9, Canada. \\ *ecabral@geofisica.unam.mx
}

\begin{abstract}
Resumen
El proceso de subsidencia en la zona metropolitana de la Ciudad de México ha sido reconocido desde principios del siglo pasado y sus efectos tienen graves consecuencias para la operación y mantenimiento de la infraestructura de la ciudad. En este trabajo se presentan las velocidades de subsidencia del terreno obtenidas de Dispersores Persistentes (Persistent Scatterers, PSs) derivados del análisis interferométrico SqueeSAR de datos ENVISAT-ASAR de Radar de Apertura Sintética adquiridos entre los años 2003 - 2010 y de nueve estaciones GPS de operación continua. A partir de estos datos se investigó la relación existente entre las velocidades de subsidencia observadas con el abatimiento del nivel estático obtenidos de hidrógrafos de 180 pozos de abastecimiento distribuidos en la zona metropolitana de la Ciudad de México. Los resultados geodésicos muestran diversos comportamientos del proceso de subsidencia en las inmediaciones de las estaciones GPS que van desde lo estable (zonas no subsidentes), zonas de baja velocidad de subsidencia, hasta zonas de muy alta velocidad de subsidencia. Las zonas de mayor velocidad de subsidencia se encuentran en regiones de gradiente horizontal de subsidencia muy bajo, dentro de la zona lacustre, con velocidades verticales de GPS tan altas como -273 mm/año. Áreas con gradientes horizontales de subsidencia locales muy altos inducen a su vez el potencial de desarrollo de grietas y fallas de tensión en la superficie. El análisis de los datos de hidrógrafos de los últimos 20 años indica que el nivel del agua subterránea ha descendido hasta $30 \mathrm{~m}$ en los casos más severos; sólo en algunos cuantos casos se registra la recuperación del nivel estático y es únicamente en aquellos pozos ubicados en áreas de baja velocidad de subsidencia. La caída de los niveles del agua subterránea se aprecia en todo el rango de velocidades de subsidencia, sugiriendo que el sistema acuífero-acuitardo se encuentra bajo severo estrés debido a la extracción extrema de agua subterránea que excede por mucho la magnitud de la recarga natural. La baja correlación entre la velocidad de subsidencia del terreno y la caída del nivel estático sugiere que existen variables adicionales que condicionan de manera importante los procesos de subsidencia, tales como la litología, la potencia del acuitardo, el contenido de agua, el comportamiento elasto-plástico de la hidroestratigrafía, la reducción de la presión de poro y el volumen de extracción. Al analizar la relación del espesor del acuitardo superior con las velocidades de subsidencia se observa una correlación positiva entre ambas variables.
\end{abstract}

Palabras clave: subsidencia, abatimiento, GPS, InSAR, Ciudad de México. 


\begin{abstract}
The process of land subsidence in the Mexico City Metropolitan Area has been recognized since the beginning of the last century and poses severe challenges for the operation and maintenance of the city's infrastructure. In this work we present land subsidence velocity results from Persistent Scatterers (PSS) through a SqueeSAR interferometric analysis of ENVISAT-ASAR Synthetic Aperture Radar data acquired during the $2003-2010$ period from nine continuous GPS stations. We then investigated the relationship between the observed subsidence rates and the groundwater level decrease obtained from 180 water well hydrographs distributed throughout the Mexico City Metropolitan Area. Geodetic results indicate differences in land subsidence in the vicinity of the GPS stations, ranging from stable (zero subsidence zones), to slow and rapid subsidence zones. The highest subsidence rates occur in sites with very low local subsidence gradients within the lacustrine sector of the city and vertical GPS velocities of up to $-273 \mathrm{~mm} / \mathrm{yr}$. Areas with very high local subsidence gradients induce the greatest hazard as they increase the potential for shallow tensile cracks and faulting. The analysis of well hydrographs over the last 20 years indicates groundwater drawdowns of up to $30 \mathrm{~m}$ in the most severe cases; groundwater level recovery occurs in very few cases, and only where well locations are within areas of very low subsidence rates. Declining groundwater levels are found throughout the entire range of subsidence values, suggesting that the aquifer-aquitard system is under severe stress due to the extreme groundwater extraction that far exceeds the magnitude of natural recharge. The low correlation between the subsidence rates and the decrease in groundwater levels further suggests that additional variables play an important role in the subsidence process, such as the lithology, the aquitard thickness, water content, the elasto-plastic behavior of the hydrostratigraphy, the drop in pore pressure and groundwater overdraft. A positive correlation is found between land subsidence rates and the thickness of the upper aquitard.
\end{abstract}

Keywords: Subsidence, groundwater withdrawal, GPS, InSAR, Mexico City.

\section{Introducción}

La subsidencia y el fallamiento superficial asociado son procesos que afectan áreas urbanas y están relacionados con la extracción intensiva del agua subterránea, lo cual genera pérdida de la presión de poro en medios granulares y conlleva a la deformación del terreno (e.g. Galloway y Hoffmann, 2007; Galloway y Burbey, 2011). Estos procesos están controlados por las condiciones locales de hidroestratigrafía, geomecánica y geología estructural y es posible medirlos con técnicas InSAR, que proveen una herramienta efectiva en costo, con cobertura espacial adecuada y resolución temporal suficiente para observar deformación a largo plazo de la zona de estudio deseada (Galloway y Burbey, 2011; Tomás et al., 2014). La Ciudad de México es conocida por estar intensamente afectada por subsidencia del terreno que resulta en una mayor vulnerabilidad a inundaciones y fallamiento superficial intenso, y que ha producido daños severos a la infraestructura durante décadas (Carrillo, 1948; CHVM, 1963; Figueroa-Vega, 1984; Mazari et al., 1992; Ortega-Guerrero et al., 1993; Lesser-Illades y GonzálezPosadas, 2005; Cabral-Cano et al., 2008, 2010).

Este trabajo tiene como objetivo correlacionar las series de tiempo de velocidades de subsidencia derivadas del análisis SqueeSAR a partir de datos del sensor Advanced Synthetic Aperture Radar (ASAR) a bordo del Environmental Satellite (ENVISAT) y de 9 estaciones GPS distribuidas en la Zona Metropolitana de la Ciudad de México (ZMCM), con los abatimientos del nivel estático observados entre 1990 y 2010 para un grupo de 180 pozos disponibles. Este análisis pretende ofrecer criterios adicionales de decisión, de manera que el proceso de subsidencia sea considerado con la debida relevancia en las políticas de administración sustentable de recursos hídricos, el uso de suelo y la mitigación de los efectos de fracturamiento superficial de tensión asociado a este proceso.

\section{Contexto geológico}

El asentamiento histórico del área urbana en la Ciudad de México se ha caracterizado por establecerse principalmente en la zona que corresponde al antiguo Lago de Texcoco, y más recientemente también ocupa espacios en la zona correspondiente al Lago de Xochimilco y de Chalco (UAM, 2005). Las tendencias actuales de ocupación urbana se caracterizan por definir los contornos urbanos previamente establecidos, lo que genera que la densidad de población aumente en las zonas de lago. Diversos autores han investigado la estratigrafía de la cuenca y su funcionamiento hidrogeológico, con estudios de campo y datos provenientes de pozos de extracción de agua, cimentaciones y pozos profundos (e.g. Arce et al., 2013; Morales-Casique et al., 2014). Los trabajos de Mooser (1975), Aguayo et al. (1989), Vázquez-Sánchez y Jaimes-Palomera (1989) y Santoyo et al. (2005) enfatizan la presencia de secuencias sedimentarias en las zonas de lago de México y Chalco, con actividad volcánica reciente y contemporánea al depósito que generan intercalaciones de rocas volcánicas con sedimentos lacustres, siendo la parte superior del sistema una capa de sedimentos ricos en arcillas. Esta unidad sobreyace a depósitos volcánicos, los cuales a su vez reposan sobre rocas calcáreas. En el proceso sedimentario contemporáneo a los depósitos volcánicos se 
generaron suelos ricos en alófano (de forma esférica de $\sim 4$ nm de diámetro) e imogolita (de forma tubular); minerales cuya presencia en la secuencia estratigráfica genera un elevado potencial de compresibilidad, lo que aunado al descenso constante de los niveles del agua subterránea se traduce en subsidencia (Carreón-Freyre et al., 2006).

Herrera et al. (1989) definen al sistema acuífero de la Cuenca de México que se constituye por 4 cuerpos con funcionamiento hidráulico diferente:

a) Acuitardo de $\sim 70 \mathrm{~m}$ de espesor de flujo esencialmente vertical. Consiste en dos grandes horizontes de arcilla separados por una capa delgada de material arenoso. Esta unidad cede agua en flujo vertical por goteo al acuífero inferior por la pérdida de presión debida a la extracción, lo que produce consolidación y reacomodo en las arcillas, generando hundimientos en la superficie.

b) Acuífero granular de $800 \mathrm{~m}$ de espesor. Esta unidad acuífera está formada por rocas volcánicas, siendo los $300 \mathrm{~m}$ superiores los de importancia para la extracción de agua en la Ciudad; se encuentra en condiciones de semiconfinamiento por el acuitardo superior. La recarga de este acuífero se da en las sierras que circundan la cuenca.

c) Acuífero volcánico de $2000 \mathrm{~m}$ de espesor, que consiste en rocas volcánicas fracturadas sobreyaciendo a las rocas carbonatadas.

d) Unidad calcárea profunda de funcionamiento incierto dentro del sistema acuífero.

\section{Interferometría de dispersores persistentes y subsidencia asociada a la extracción de agua subterránea usando InSAR}

Una de las aplicaciones de técnicas InSAR englobadas como PSInSAR o Persistent Scatterers Interferometry, que utilizan el principio de Dispersores Persistentes o PSs (Persistent Scatterers; Ferretti et al., 2001, 2011), es detectar variaciones temporales en la elevación del terreno. La solución para maximizar el número de observaciones a lo largo del tiempo depende de puntos correlacionables, usualmente en conjuntos de por lo menos 15 escenas de Radar de Apertura Sintética (Synthetic Aperture Radar, SAR). Se utilizan elementos dentro de la escena SAR que conservan su coherencia en la fase de la señal a lo largo de todas las escenas utilizadas. A estos elementos se les llama Dispersores Permanentes o Persistentes, comúnmente conocidos como PSs. El número de estos dispersores aumenta sustancialmente en áreas urbanas debido a que mantienen sus características a lo largo del tiempo, aunque la densidad de estos elementos es variable según las características del terreno y tipo de estructuras y aditamentos urbanos. La aplicación de esta técnica depende de la selección de una imagen maestra a partir de la que se correlacionan el resto de las escenas SAR y se detectan los elementos congruentes en todas ellas (Ferretti et al., 2001). Los puntos PSs provienen de objetivos diversos (cruces de calles, antenas, edificios, etc.), por lo tanto, la densidad de PSs en zonas urbanas es alta pero también variable, mientras que en regiones no urbanizadas la densidad de PSs es mucho menor y en ocasiones es posible que se llegue a obtener muy poca o incluso nula información. Una segunda generación de técnicas PSInSAR es SqueeSAR, que además de identificar los PSs, hace uso también de Dispersores Distribuidos (Distributed Scatterers, DS). Los DS provienen principalmente de afloramientos dispersos, escombros, áreas no cultivadas y zonas de baja cobertura vegetal. Esta técnica proporciona información adicional en regiones homogéneas de baja reflectividad y mejoran la densidad de datos de las nubes de PSs (Ferretti et al., 2011).

En el caso de la ZMCM, hemos correlacionado la cartografía de la subsidencia con datos hidrogeológicos para mejorar nuestro conocimiento de este proceso, con el fin de generar una herramienta para la administración del recurso hídrico subterráneo (e.g. Hernández-Espriú et al., 2014) y proponer medidas de mitigación de la subsidencia causada por el abatimiento del nivel estático de los sistemas acuíferos. Una limitante de la medición con métodos tradicionales de la subsidencia del terreno radica en la amplia extensión de las regiones sujetas a cambios de elevación, su alto costo, y la necesidad de emplear bancos de nivel o puntos fijos de referencia que no estén afectados por el proceso de subsidencia o la subestimación resultante en el amarre de estaciones en zonas de alta velocidad de subsidencia (e.g. Cabral-Cano et al., 2008). Las aplicaciones de las técnicas basadas en análisis SAR han ayudado a mejorar la caracterización de la respuesta espacio-temporal de los acuíferos a cambios hidromecánicos inducidos por el cambio de almacenamiento hidrogeológico (Galloway y Hoffmann, 2007); así mismo, la observación de los movimientos en superficie proporcionan una guía para la evaluación de los modelos de recarga y descarga subterránea, identificación de barreras subterráneas, caracterización de fluctuaciones estacionales del terreno y las relaciones de variación en el comportamiento extractivo.

\section{Métodos y materiales}

\subsection{Datos SqueeSAR y GPS}

El mapa de velocidades de subsidencia usado en este trabajo (Solano-Rojas et al., 2012) fue generado por el método SqueeSAR (Ferretti et al., 2011). Este conjunto de datos consiste en una nube de puntos obtenidos a partir de 29 escenas SAR del sensor ENVISAT-ASAR adquiridas entre 2003 y 2010, a partir de las cuales se analizó la subsidencia en la Ciudad de México. Para este proceso se utilizó la escena del 29 de julio de 2005 como escena maestra.

Además de la nube de puntos SqueeSAR, se analizaron series de tiempo de 9 estaciones GPS continuas en el área de estudio. Cuatro de ellas están localizadas dentro del área lacustre de mayor subsidencia (MOCS, MPAA, MRRA y 
UPEC) y el resto en áreas estables (Figura1a). Las series de tiempo de las estaciones GPS fueron generadas a partir de las soluciones diarias no-fiduciales de coordenadas utilizando la estrategia de Posicionamiento de Punto Preciso (Zumberge et al., 1997), que incluyen consideraciones de retrasos hidrostáticos y húmedos a partir de parámetros del Vienna Mapping Function (VMF1), correcciones de centro de fase dependientes del azimut y elevación de archivos IGS08 ANTEX (disponibles vía FTP en sideshow.jpl.nasa. gov), y correcciones por cargas de mareas (http://holt.oso. chalmers.se). Las estimaciones de posición diarias nofiduciales se transformaron al marco de referencia IGS08, que es conforme con la realización de ITRF2008 (Altamimi et al., 2011) usando una transformación de Helmert de siete parámetros.

La estación UCHI fue seleccionada como sitio de referencia para ajustar el nivel de referencia de los mapas de velocidades generados por la nube de puntos PSs. El algoritmo SqueeSAR utilizado, desarrollado por TeleRilevamento Europa (TRE, 2011), inicia seleccionando PSs detectables en todos los interferogramas, desenvolviendo sus cambios de fase a través del tiempo. Estos PSs son filtrados para detectar y remover la contribución atmosférica de fase, conocida comúnmente como APS (atmospheric phase screen). Los factores que influyen las mediciones de fase de InSAR son: la magnitud de la deformación de la señal de interés, la contribución topográfica de fase o error del modelo digital de elevación y la contribución orbital debido al valor de incertidumbre en las órbitas satelitales (que se minimiza con el uso de órbitas de satélites precisas), la contribución de fase debida a la fase atmosférica de la escena, la fase por dispersión (que puede ser ignorada debido a que los PSs por su naturaleza no cambian en el tiempo) y el ruido por efecto de fase que puede ser inadvertido por la congruencia de los PSs en las imágenes. A lo largo de este trabajo, se utilizan directamente las series de tiempo de la nube de puntos SqueeSAR proyectadas en la componente vertical para analizar las variaciones en el tiempo de los movimientos del terreno.

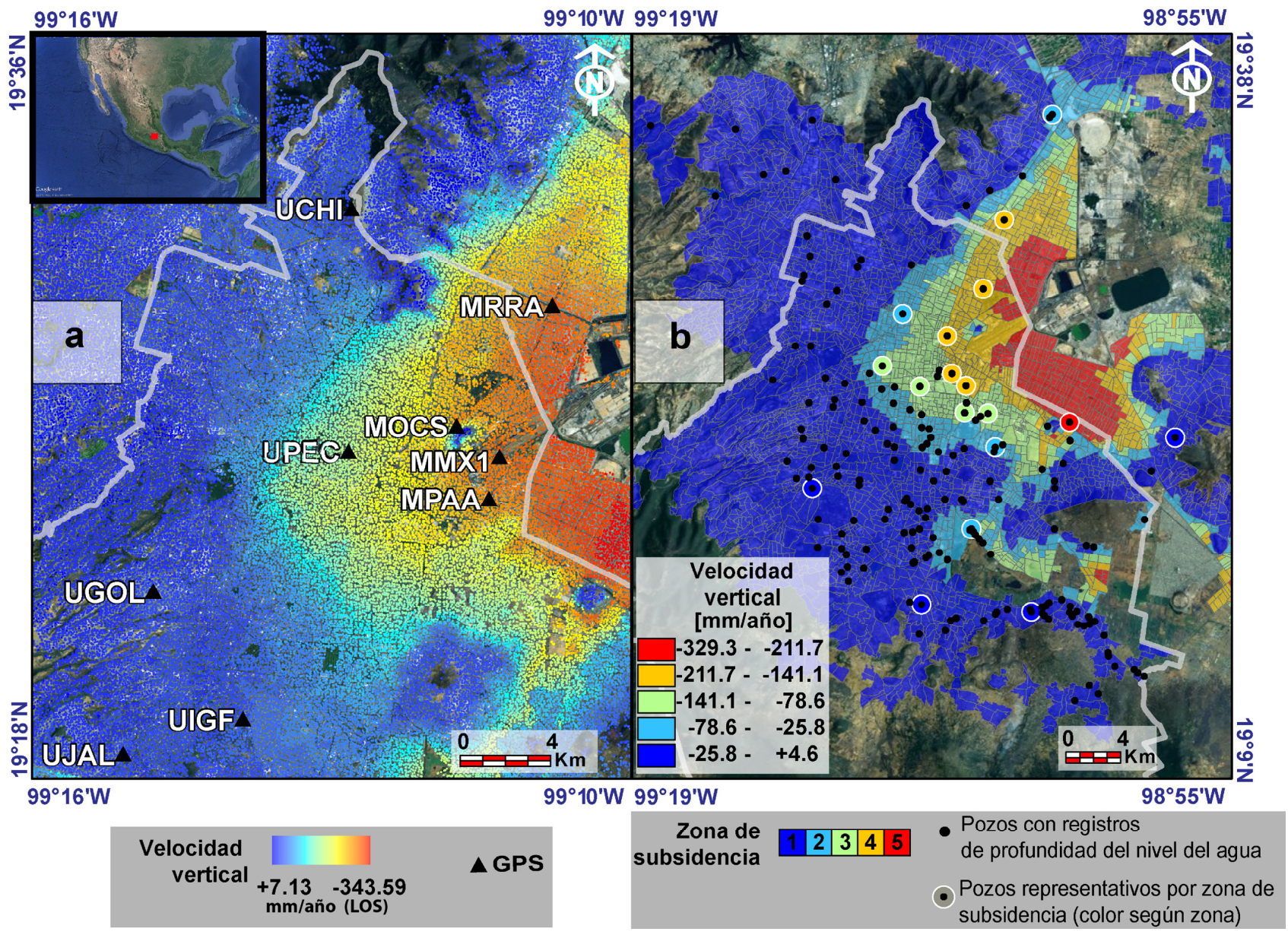

Figura 1. a) Localización de la Ciudad de México. Nube de puntos PSs detectada por medio del procesamiento SqueeSAR de velocidades de subsidencia en LOS (Line of Sight). Los triángulos de color negro muestran la ubicación de las estaciones GPS en el área de estudio; b) Zonificación de las AGEBs por velocidad de subsidencia y localización de los 180 pozos de extracción de agua utilizados en este estudio, así como los 17 representativos de las zonas de subsidencia. 
4.2. La relación de subsidencia GPS - InSAR y abatimiento de nivel estático en pozos

A partir de la información de 9 estaciones de GPS (Figura 1a), se realizaron comparaciones de las series de tiempo de desplazamiento del terreno en la componente vertical y de los PSs en una zona de influencia de hasta $300 \mathrm{~m}$ de radio a partir de la posición de cada una de las estaciones GPS. Dicha zona de influencia asegura la presencia de PSs para todas las estaciones analizadas y provee información de la deformación en los alrededores de las mismas. La abundancia de retrodispersores permanentes PSs en las inmediaciones de cada estación de GPS varía debido a que la existencia de puntos PSs depende de la persistencia de coherencia a lo largo de las escenas SAR $\mathrm{y}$ de otros factores previamente mencionados. Las gráficas resultantes se presentan en la Figura 2. En esta figura, las observaciones derivadas de GPS y de PSs consideradas como representativas del mismo sitio, son congruentes cuando las pendientes en las gráficas respectivas son similares a pesar de que no se traslapen, debido a que sus puntos de inicio/fin de la medición de deformación pueden variar en tiempo. En ambos casos las pendientes de las series de tiempo GPS y de cada PS representan la velocidad de subsidencia.

Asimismo, a partir de la información disponible de pozos de extracción de agua subterránea (SACM, 2006; CONACyT-ICyTDF, 2012) se seleccionaron aquellos pozos cuya información histórica del nivel potenciométrico del sistema acuífero de la Ciudad de México presentan mayor consistencia, es decir, aquellos que presentan varias observaciones, con una tendencia claramente definida. Con este criterio, se seleccionaron 180 pozos, de los cuales, se obtuvo además la tasa de abatimiento para cada serie de tiempo, considerando un período temporal de 1990 a 2010. En los pozos para los cuales no se contaba con mediciones para el año 2010, se calculó el valor estimado con una regresión lineal, de la que se consideró la pendiente como

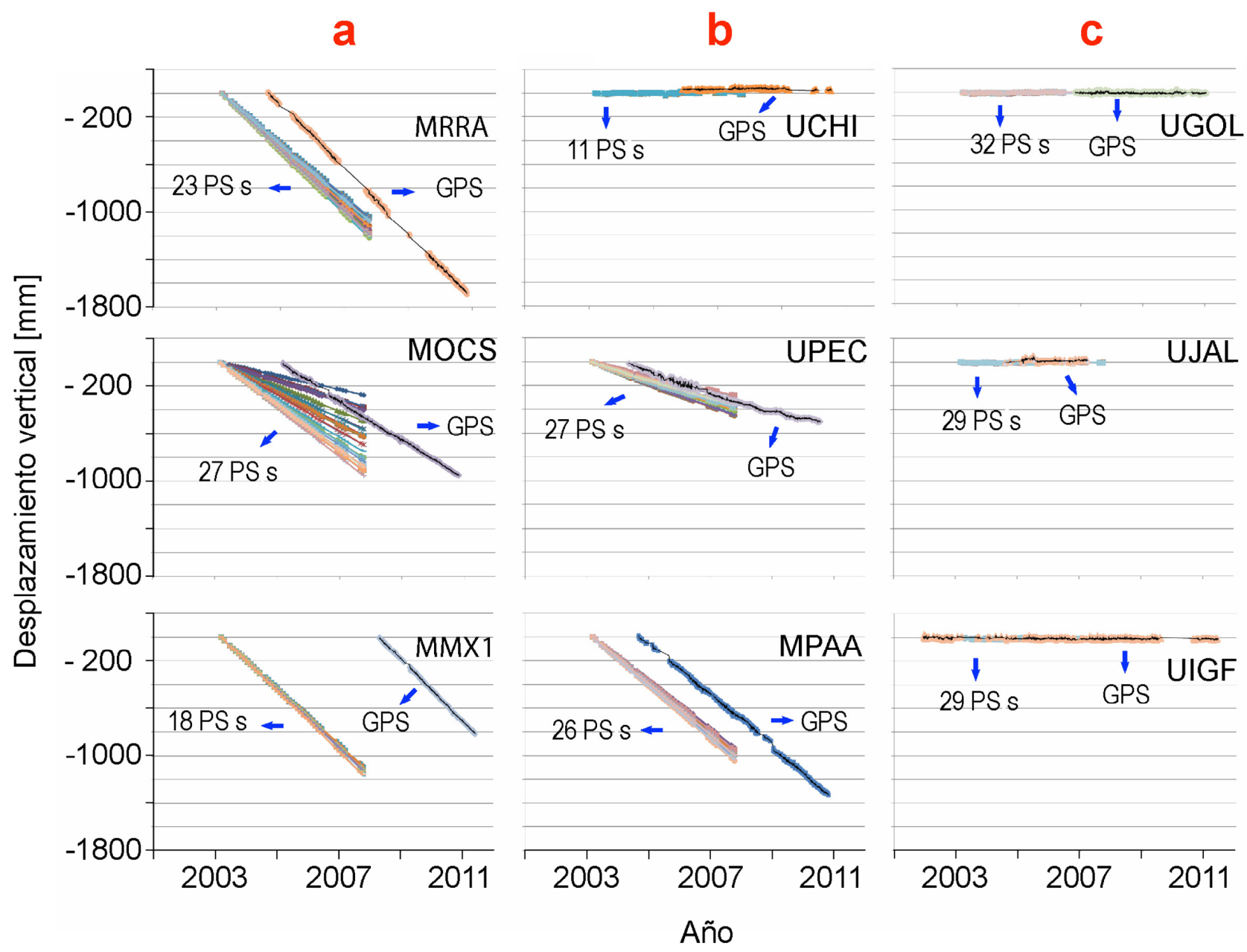

Figura 2. Series de tiempo de nueve estaciones GPS en el área de estudio y los correspondientes PSs en un radio de $300 \mathrm{~m}$. Las pendientes de las series de tiempo representan las velocidades de subsidencia. La descripción detallada de cada estación se encuentra en el texto; a) Estaciones MRRA, MOCS y MMX1 se ubican en zonas de alta tasa de subsidencia. La subsidencia es diferencial en los alrededores de MOCS y MRRA, como lo muestran las series de tiempo derivadas de los PSs; b) UCHI se encuentra en una zona estable, UPEC y MPAA se ubican en zonas subsidentes y también se observa subsidencia diferencial en sus alrededores; c) UGOL, UJAL y UIGF se ubican en zonas estables y muestran desplazamiento casi nulo. 
la tasa de abatimiento.

Con el fin de agrupar sistemáticamente los valores de abatimiento en pozos y facilitar su correlación y posterior análisis de vulnerabilidad, se generó una zonificación de la velocidad de subsidencia utilizando las Áreas Geoestadísticas Básicas (AGEBs) del Instituto Nacional de Estadística y Geografía (INEGI) correspondientes al Distrito Federal y el Estado de México (INEGI, 2011) dentro de la zona de cobertura de los datos de InSAR. Dichas zonas sirven para fines estadísticos y administrativos para varios órganos gubernamentales y tienen su origen en la distribución de la traza urbana. Para ello, se promedió el valor de velocidad de subsidencia de los puntos de la nube SqueeSAR para cada polígono de AGEB. El resultado de dicha zonificación se aprecia en la Figura 1b. También en esta figura se muestra la ubicación de los 180 pozos con registros de abatimientos, así como de 17 pozos representativos que se utilizaron para comparar las series de tiempo de abatimiento y subsidencia, eligiendo para este propósito los PSs en un radio de $150 \mathrm{~m}$ alrededor de cada pozo, ya que la intención es observar la deformación en las proximidades de los pozos. En la zona 1 (de muy baja subsidencia) la abundancia de pozos con históricos disponibles (y de pozos en operación) es mayor y disminuye la cantidad de pozos disponibles hacia la zona 5 (de muy alta subsidencia), en donde solamente hay un pozo disponible.

4.3. Relación espesor del acuitardo-velocidad de subsidencia

A partir de datos de la distribución espacial de los espesores de los depósitos lacustres en 372 pozos de la Ciudad de México, obtenidos de SACM (2006), se realizó un análisis comparativo de las velocidades de subsidencia derivadas de PSs con los correspondientes espesores del acuitardo, con el objetivo de generar un modelo lineal simple que pudiera predecir los espesores asociados a cada velocidad de subsidencia y/o viceversa.

\section{Resultados y Discusión}

\subsection{Relación de subsidencia GPS - InSAR}

Las gráficas comparativas de series de tiempo de GPS y de PSs, distribuidos en un radio de $300 \mathrm{~m}$ a partir de cada estación continua GPS, se muestran en la Figura 2. Las mediciones de GPS y de PSs presentan un comportamiento prácticamente lineal. La pendiente de cada serie de tiempo representa la velocidad de subsidencia registrada para ese punto: la congruencia y menor dispersión en las pendientes de las series de tiempo de los PSs indican que, para cada estación GPS en particular, los puntos circundantes tienen componentes de movimiento vertical similares. Las series de tiempo de los PSs que muestran dispersión, en forma de abanicos, son el resultado de que los puntos PSs, cercanos a la estación GPS, tienen variabilidad en sus velocidades de subsidencia por estar dentro de una zona de mayor gradiente horizontal. Esta condición es el resultado de las características estratigráficas y estructuras geológicas del subsuelo que generan velocidades de subsidencia diferenciales que, a su vez, producen fallamiento diferencial en la superficie (Cabral-Cano et al., 2010).

En la Figura 1a se observa la ubicación de cada estación de GPS con los PSs circundantes. Las estaciones MRRA, MOCS y MMX1 (Figura 2a) se encuentran en zonas de alta velocidad de subsidencia, con desplazamientos verticales entre -160 y $-273 \mathrm{~mm} /$ año. Se observa que los 18 PSs circundantes a la estación MMX1, que tiene una velocidad de subsidencia de $-262 \mathrm{~mm} / \mathrm{año}$, presentan un comportamiento de entre -222 y $-253 \mathrm{~mm} / \mathrm{año}$, lo que provoca el traslape de sus series de tiempo. En la estación MRRA, con velocidades verticales de la estación GPS de -273 mm/año, se puede apreciar que las velocidades de los puntos PSs forman un ligero abanico en sus series de tiempo, con velocidades de -225 a $-264 \mathrm{~mm} / \mathrm{año}$. En la estación MOCS es evidente que el comportamiento de los 27 PSs circundantes a la estación de GPS generan un abanico abierto, con velocidades de subsidencia que varían de -60 hasta -207 mm/año (comparadas con la velocidad de -160 $\mathrm{mm} /$ año de la estación GPS) y que generan esfuerzos que producen fallamiento superficial de tensión. El fenómeno de fallamiento en áreas de alto gradiente horizontal de subsidencia tiene consecuencias en la infraestructura urbana $\mathrm{y}$ ha sido evaluado recientemente también en términos del incremento de la vulnerabilidad acuífera de la Ciudad de México, bajo la metodología de DRASTIC-Sg (HernándezEspriú et al., 2014). Esta situación se debe a que MMX1 y MRRA se encuentran ubicadas en la zona de lago, mientras que MOCS se encuentra cercano al piedemonte del Peñón de los Baños, provocando que los puntos PSs dentro de un radio de $300 \mathrm{~m}$ muestren velocidades tanto de la estructura volcánica del Peñón de los Baños (cuya deformación es nula), como de la zona de lago, cuya subsidencia es cercana a los $-207 \mathrm{~mm} /$ año. Ésta es una combinación de factores que genera la aparición de fallamiento superficial asociado.

La Figura 2 b muestra que las observaciones en la estación UCHI corresponden a una deformación prácticamente nula, tanto en el GPS (-0.3 mm/año) como en los PSs (-1.62 a $+0.52 \mathrm{~mm} / \mathrm{año})$. En las inmediaciones de la estación UPEC se aprecia una mayor velocidad de subsidencia (-75.7 mm/año) y los puntos PSs aledaños presentan una dispersión en sus velocidades, con valores de -62 y -98 mm/año, mientras que en la estación MPAA se observa una velocidad de subsidencia de $-220.2 \mathrm{~mm} / \mathrm{año}$, comparada con velocidades de subsidencia desde -202 hasta -227 mm/año obtenidas con los PSs.

La Figura 2c muestra las gráficas de las estaciones UGOL, UJAL y UIGF, que comparten con UCHI la característica de encontrarse en zonas estables y con subsidencia prácticamente nula, observada tanto en las mediciones de GPS como en los PSs. Es apreciable el 
traslape de las series de tiempo, no obstante, estas zonas no presentan variabilidad significativa de la velocidad de subsidencia y por lo tanto tampoco se espera la aparición de gradiente horizontal en el área circundante.

\subsection{Relación desplazamiento del terreno - nivel del agua subterránea}

Las gráficas resultantes por cada zona de subsidencia se muestran en la Figura 3. La zona 5 representa la zona del área metropolitana con mayor velocidad de subsidencia mientras que la zona 1 no presenta subsidencia. La escala de colores empleada es la misma que en el mapa de clasificación por velocidad de subsidencia mostrada en la Figura 1. En negro se muestran las series de tiempo obtenidas de los PSs en un radio de $150 \mathrm{~m}$ alrededor de cada pozo de abastecimiento en las diferentes zonas de subsidencia. La combinación de las series de tiempo, de abatimiento y de PSInSAR, permitió diseñar gráficas de dos ejes, con valores de profundidad del nivel estático medios y valores de subsidencia del terreno en la componente vertical.

En la Figura 3, se muestra la única serie de tiempo de abatimiento para la zona 5 y las cinco series de tiempo disponibles para la zona 4. Las series son completas excepto para dos de ellas que carecen de mediciones previas a 2006, y en la que la tasa de abatimiento registrada es la menor del grupo. La Figura 3 muestra también los comportamientos registrados en las zonas de baja velocidad de subsidencia. Los correspondientes a la zona 3 muestran congruencia en las mediciones de la profundidad del nivel potenciométrico con variaciones menores de recuperaciones y abatimientos, pero siguiendo la tendencia general de abatimientos del nivel del agua, al igual que las series de tiempo para la zona 2. En el caso de la zona 1 las tendencias se vuelven menos evidentes, las etapas de recuperación y abatimientos se extienden en tiempo, aunque se aprecian tendencias de
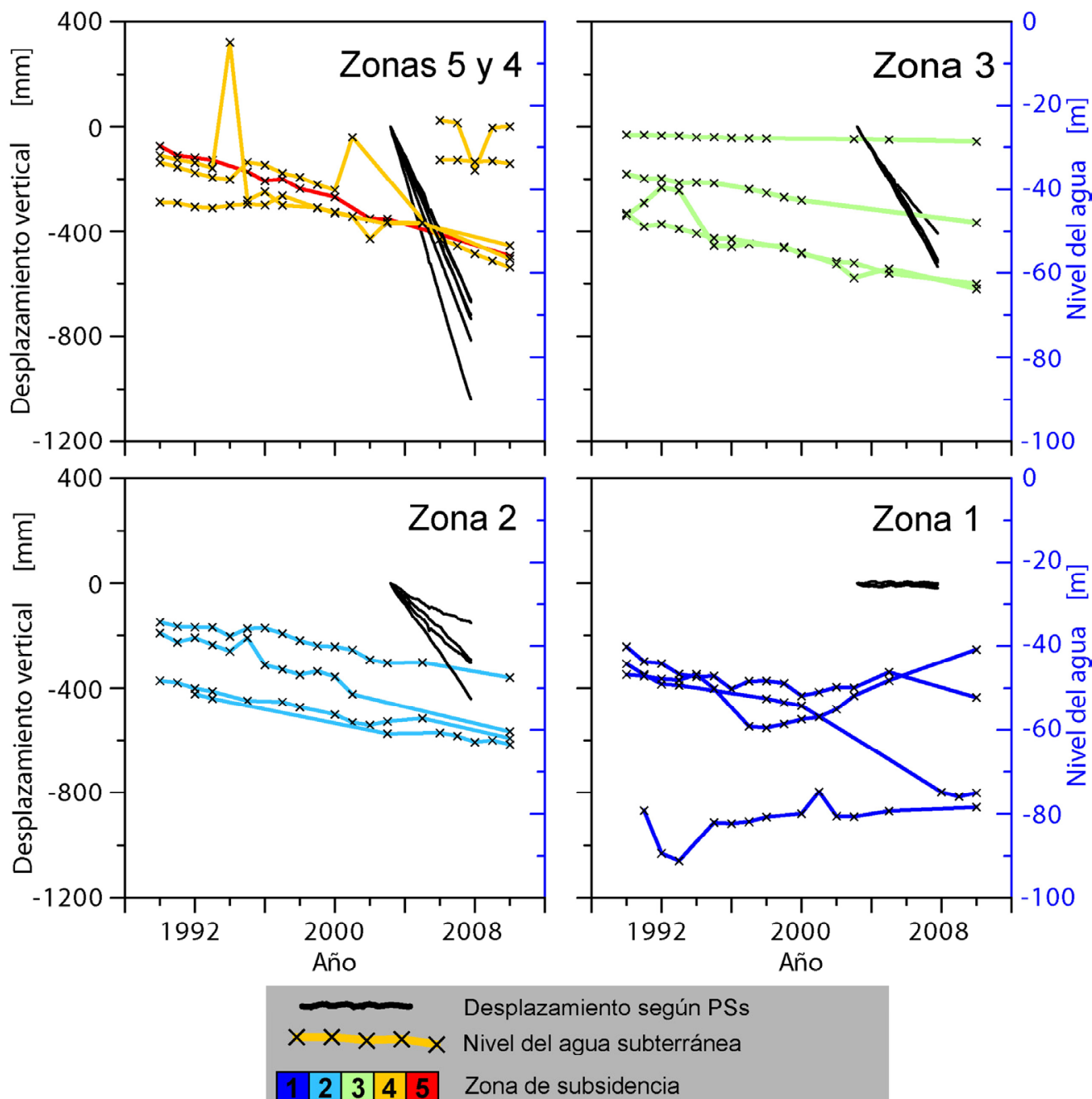

Figura 3. Series de tiempo del desplazamiento del terreno relativo al inicio de las mediciones provenientes de PSs y del nivel del agua subterránea en pozos de abastecimiento. El eje vertical en color negro corresponde con los históricos de subsidencia también en color negro. El eje vertical en color azul corresponde con los históricos de abatimientos con el código de color usado en la Figura 1. Se muestra que las tasas de abatimientos se mantienen con pérdidas consistentes del nivel del agua y sin variaciones sustanciales a lo largo de 20 años en las zonas 2,3 , 4 y 5 , congruentes con las tasas de subsidencia. 
recuperación del nivel potenciométrico.

También se observa que las tendencias de abatimientos para las zonas 5 y 4 de alta subsidencia (Figura 3) son muy similares, con abatimientos cercanos a los $30 \mathrm{~m}$, salvo para las dos series sin datos previos al 2006. En la zona 3 se observan abatimientos cercanos a los $20 \mathrm{~m}$. Para la zona 2 , se observan abatimientos de 15 a $22 \mathrm{~m}$ y en la zona 1 destacan las recuperaciones del nivel en periodos de tiempo cortos y a largo plazo, aunque también se aprecian abatimientos cercanos a los $30 \mathrm{~m}$. Estas tendencias sugieren que las tasas de abatimientos en los pozos representativos por zona de subsidencia se mantienen con pérdidas consistentes de su nivel estático y sin variaciones sustanciales a lo largo de 20 años en las zonas de mediana y alta subsidencia, y que la única excepción es la que presentan un par de pozos localizados dentro de la zona 1 de baja subsidencia, que muestran una recuperación de su nivel. Por otra parte también se observa que la tasa de abatimiento no presenta una relación lineal con la velocidad de subsidencia.

Adicionalmente, se elaboró una gráfica en donde se compara la tasa de abatimiento y la velocidad de subsidencia para cada uno de los pozos con información histórica de la profundidad del nivel del agua subterránea de los 180 pozos seleccionados y los pozos representativos por zona de subsidencia (Figura 4). La Figura 4 muestra que la gran mayoría de los pozos se mantienen con abatimientos de sus niveles estáticos y únicamente aquellos que muestran recuperación son los que se encuentran en las zonas 1 y 2 de baja velocidad de subsidencia. No se encontró ningún pozo ubicado en las zonas $3-5$, de media a alta subsidencia, que presente recuperación en su nivel estático. En esta gráfica se identifican dos pozos de la zona 4 sin datos previos al
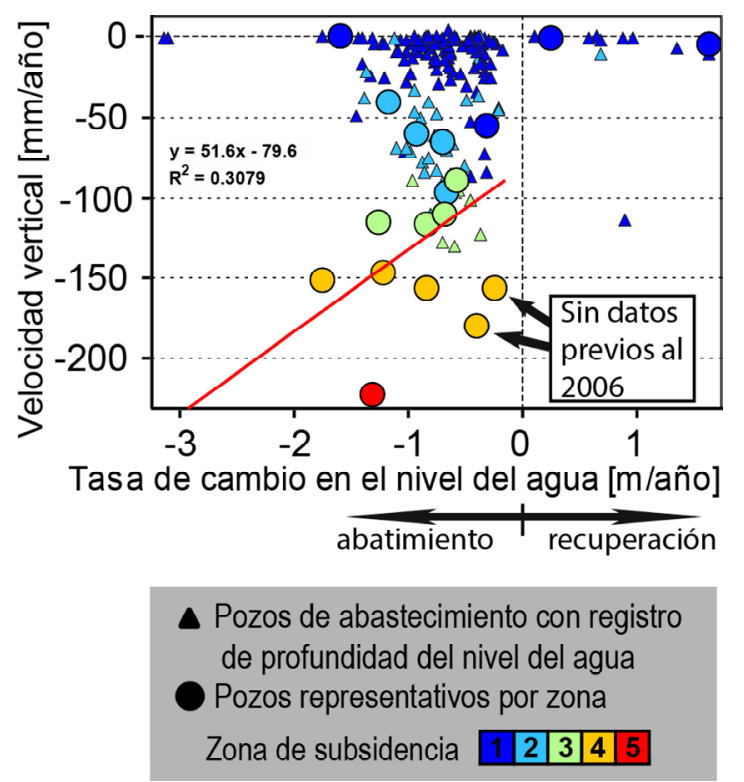

Figura 4. Comparativo de tasas de abatimiento y velocidades de subsidencia en los pozos seleccionados de la zona de estudio. La línea de color rojo representa el ajuste lineal de los valores correspondientes a las zonas 3, 4 y 5 , excluyendo los valores sin datos previos al 2006.
2006, que a pesar de su corto registro, también sugieren abatimientos del nivel del agua. Por otra parte, los intervalos de tasa de abatimiento se mantienen dentro de 0.2 a 1.8 $\mathrm{m} / \mathrm{año}$ independientemente de su ubicación en zonas de subsidencia. A partir de las velocidades de subsidencia y las tasas de abatimiento de los pozos correspondientes a las zonas 3,4 y 5 , y excluyendo los puntos sin datos previos al 2006 ( 15 elementos en total) se obtuvo un modelo lineal para representar la relación subsidencia-abatimiento. El coeficiente de correlación obtenido para este modelo es de 0.307 , debido a la dispersión de los valores medidos. El modelo obtenido se marca con una recta en color rojo en la Figura 4, y muestra una relación lineal directa entre abatimiento y subsidencia, con $-51.6 \mathrm{~mm}$ de desplazamiento vertical por año por cada metro de abatimiento del nivel del agua. Los puntos considerados son representativos de áreas puntuales, que podrían ser mejor interpretadas con un conjunto de datos más grande para analizar comportamientos locales, aunque la tendencia general está bien representada para las zonas subsidentes. Dicha relación no es observable en las zonas menos subsidentes (zonas 1 y 2, excluidas para el modelo).

A falta de un registro de la variación espacial del caudal de extracción en cada uno de los pozos estudiados, consideramos que estas observaciones son indicativas de que el proceso de subsidencia está controlado en buena medida por la estratigrafía y en particular por el contenido y espesor de arcillas. Figueroa-Vega (1984) estima que tres cuartas partes de la subsidencia en la Ciudad de México se correlacionan con la consolidación del acuitardo arcilloso y sólo una cuarta parte se debe a la compresión de materiales que conforman el acuífero. Considerando que las características elasto-plásticas de la hidroestratigrafía local permiten la consolidación y reacomodo de las partículas de arcillas de la capa superior al incrementar las tensiones efectivas como consecuencia de la extracción de agua y que el acuitardo superior cede agua en flujo vertical al acuífero inferior semiconfinado, cuando disminuye la presión de poro (Herrera et al., 1989), es posible sugerir que los abatimientos regionales influyen de manera determinante en la subsidencia, más allá de los abatimientos locales, pues generan que las zonas potentes del acuitardo cedan agua y se acumule la deformación en la columna de arcillas. Es también necesario considerar que las áreas que presentan mayor subsidencia no necesariamente se correlacionan con las mayores tasas de abatimiento en pozos, pero sí se correlacionan directamente con el espesor del acuitardo arcilloso (Cabral-Cano et al., 2008) y su consolidación al ceder agua debido a la sobreextracción del sistema, aunque dicha relación varía espacialmente.

\subsection{Análisis del espesor del acuitardo-velocidad de} subsidencia

De acuerdo con datos de 372 pozos recopilados en SACM (2006), se muestra en la Figura 5 la distribución 
espacial de los espesores de los depósitos lacustres en el área dentro de los límites políticos del Distrito Federal. Dicha distribución al ser comparada con las velocidades de subsidencia del terreno (Figura 1a), muestra que los valores más altos de velocidad de subsidencia se presentan en las áreas con los mayores espesores de los sedimentos lacustres. Para este estudio comparamos los valores de velocidad de subsidencia de la nube de puntos PSs (Figura 1a) con el espesor de los sedimentos lacustres correspondiente para 105 de nuestro conjunto de 180 pozos selectos de la Figura $1 \mathrm{~b}$ (SACM, 2006, CONACyT-ICyTDF, 2012), obteniendo así la Figura 6. Para dicho conjunto de datos se sobrepone el ajuste de mínimos cuadrados del total de los datos con una curva de primer orden. En la gráfica se observa una relación directa entre las velocidades de subsidencia y el espesor de los sedimentos lacustres, obteniendo un coeficiente de correlación de 0.52 .

Considerando que nuestro modelo está acotado a las zonas subsidentes y tomando en cuenta las condiciones previamente mencionadas, éste indica que por cada metro de sedimentos lacustres, la velocidad de subsidencia observada se incrementa en $2.59 \mathrm{~mm} /$ año.

Ortiz-Zamora y Ortega-Guerrero (2010) observan que en la zona de Chalco (en las vecindades del sistema acuífero, al este de nuestra zona de estudio) en donde el acuífero tiene espesores menores a $100 \mathrm{~m}$, las pérdidas de presión de poro juegan un papel importante en el flujo subterráneo y en la $99^{\circ} 19^{\prime} \mathrm{W}$

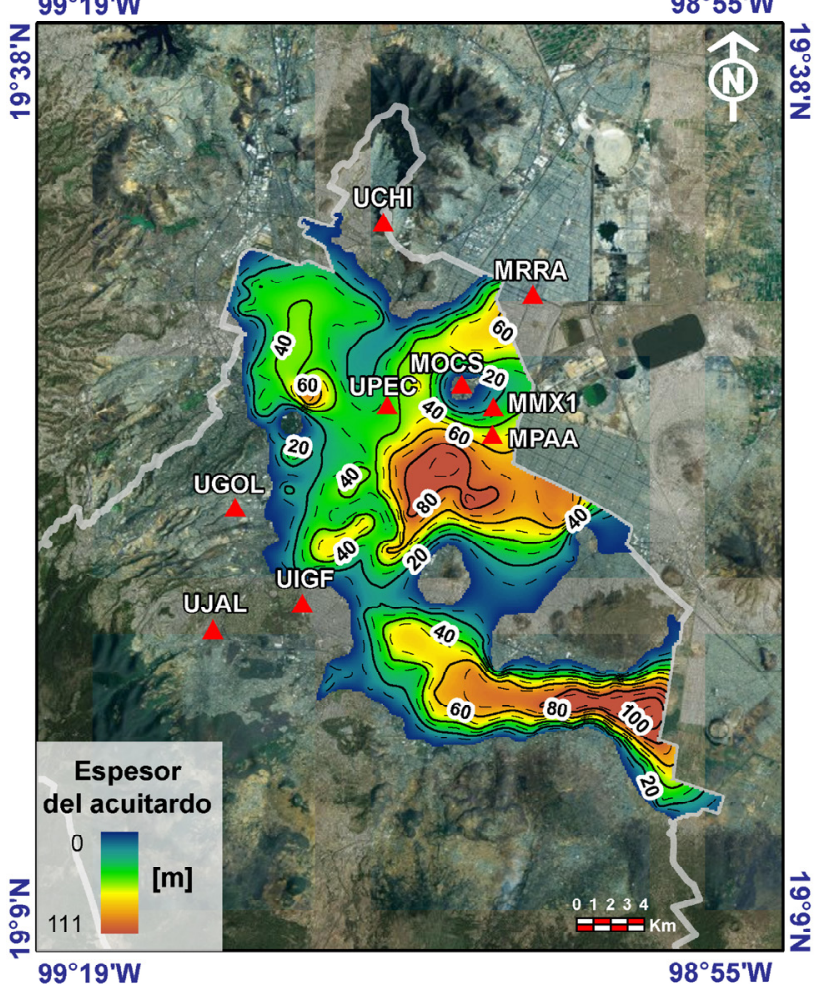

Figura 5. Distribución espacial de los espesores del acuitardo superior de acuerdo con datos provenientes de 372 pozos (SACM, 2006), conformado por depósitos lacustres de baja permeabilidad. generación de fracturas por lo que deben ser monitoreadas y tomadas en cuenta para la planeación urbana futura considerando el crecimiento del mismo.

Ortega-Guerrero et al. (1999) y Ortiz-Zamora y OrtegaGuerrero (2010) discuten acerca del papel que juega la litología y el espesor del acuitardo superior en el proceso de subsidencia y presentan un modelo para predecir la subsidencia total acumulada para espesores de hasta $300 \mathrm{~m}$ en la zona de Chalco, donde las tasas de abatimiento son de $\sim 1.5 \mathrm{~m} /$ año y velocidades de subsidencia de $400 \mathrm{~mm} /$ año. Para probar la exactitud de nuestras estimaciones, aplicamos nuestros modelos para obtener valores de espesor y tasas de abatimiento a partir de la velocidad observada en dicha zona (-400 $\mathrm{mm} / \mathrm{año}$ ) obteniendo un espesor del acuitardo teórico de $\sim 173 \mathrm{~m}$ y una tasa de abatimiento de $6.2 \mathrm{~m} / \mathrm{año}$. Esto nos habla de la heterogeneidad del medio y refuerza la necesidad de generar modelos locales a partir de los datos con los que se cuenta de subsidencia, espesor y abatimiento.

\section{Conclusiones}

El análisis comparativo de series de tiempo derivadas de GPS y de puntos PSs en este trabajo, muestra que la distribución espacial de la magnitud de subsidencia dentro de la ZMCM se mantiene dentro de los mismos parámetros que aquellos realizados previamente, utilizando sensores SAR diferentes (Cabral-Cano et al., 2008), a la vez que proporcionan una herramienta para medir deformación de la superficie con alta densidad espacial.

El análisis de las tendencias de abatimiento de los niveles potenciométricos de la zona de estudio muestra que existe una relación entre altas velocidades de subsidencia

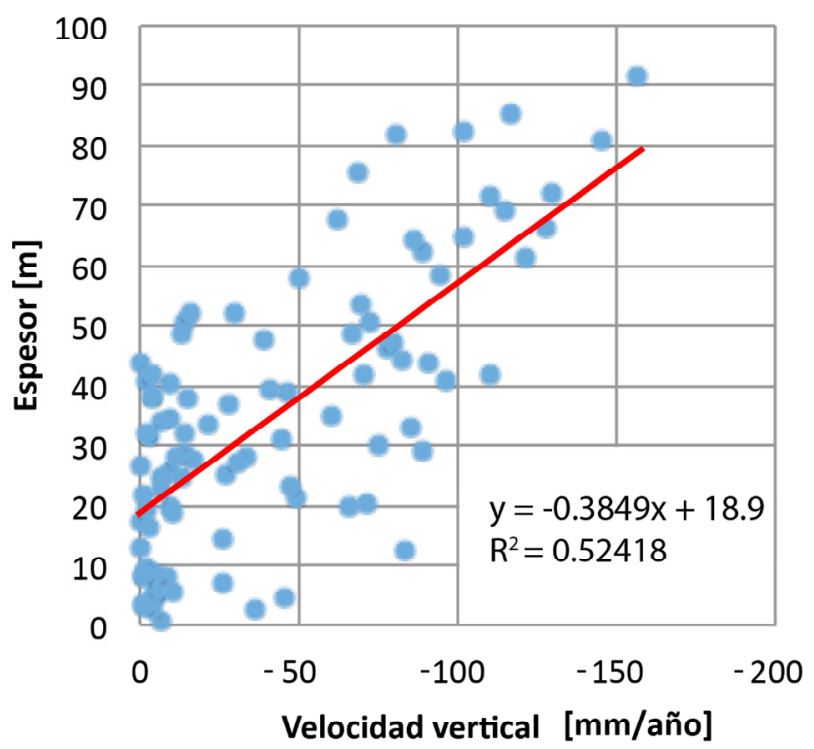

Figura 6. Gráfica comparativa de la velocidad de subsidencia y su respectivo espesor del acuitardo superior. La línea de color rojo representa el ajuste lineal de los pares graficados. 
y altas tasas de abatimiento. Esto es, en las zonas de mayor subsidencia las tasas de abatimiento son altas también, pero esta condición no se presenta en el caso inverso. Las altas tasas de abatimiento no son exclusivamente correlacionables con altas velocidades de subsidencia, sino que se presentan asimismo en zonas de baja subsidencia. Por otra parte, los escasos registros de pozos con recuperación en sus niveles estáticos sólo se presentan en las zonas de baja subsidencia, lo que sugiere un elemento a evaluar si se diseña una estrategia de mitigación del proceso de subsidencia y conservación del acuífero, y acuitardo en la Ciudad de México. Consideramos que la relación subsidencia-abatimiento presenta estas características ya que la distribución de los espesores de arcillas en el sistema acuífero no es uniforme, además de que el comportamiento puede estar también controlado por el caudal efectivo de extracción en cada pozo. Por lo tanto, las tendencias regionales y locales de abatimiento generan un comportamiento drástico de subsidencia del terreno en las regiones donde el acuitardo tiene mayor espesor. Estos argumentos sugieren que además de la sobre-extracción del agua subterránea, existen variables adicionales que condicionan de manera importante los procesos de subsidencia, tales como la litología, la potencia del acuitardo, el contenido de agua, el comportamiento elastoplástico de la hidroestratigrafía, la pérdida de la presión de poro y el volumen de extracción. Nuestras observaciones muestran que la velocidad de subsidencia está relacionada tanto con el abatimiento del nivel freático como con el espesor del acuitardo superior, aunque no limitadas a estos dos parámetros y sólo el análisis detallado en zonas bien definidas podría proveer de modelos con mayor precisión

\section{Agradecimientos}

Esta investigación se apoyó en la infraestructura geodésica instalada como parte de los proyectos UNAM PAPIIT IN109315-3, IN108611 e IN104213-2. Agradecemos a la National Aeronautics and Space Administration (NASA) de los EUA por el apoyo a través del proyecto NNX12AQ08G, al proyecto UNAM PAPIIT IV100215, al CONACyT por medio del Proyecto FOMIX 121128 y al ICyTDF, actualmente Secretaría de Ciencia, Tecnología e Innovación del Distrito Federal por sus diversos apoyos. Agradecemos también al European Space Agency (ESA) por permitir el uso de las escenas SAR a través del proyecto ESA CAT-1 1409 y por las adquisiciones directas de TeleRilevamento Europa (TRE).

El primer autor fue becario PAPIIT y es actualmente becario del CONACyT y del programa COMEXUS Fulbright-García Robles.

\section{Referencias}

Aguayo, J., Marín, S., Sánchez, F., 1989, Evolución geológica de la Cuenca de México, en Simposio sobre tópicos Geológicos de la Cuenca del Valle de México: Sociedad Mexicana de Mecánica de Suelos, México, D.F., 25-41.

Altamimi, Z., Collilieux, X., Métivier, L., 2011, ITRF2008: an improved solution of the international terrestrial reference frame: Journal of Geodesy, 85, 457-473.

Arce, J., Layer, P., Morales-Casique, E., Benowitz, J., Rangel, E., Escolero, O., 2013, New constraints on the subsurface geology of the Mexico City Basin: The San Lorenzo Tezonco deep well, on the basis of ${ }^{40} \mathrm{Ar} /{ }^{39} \mathrm{Ar}$ geochronology and whole-rock chemistry: Journal of Volcanology and Geothermal Research, 266, 34-49.

Cabral-Cano, E., Dixon, T. H., Miralles-Wilhelm, F., Díaz-Molina, O., Sánchez-Zamora, O., Carande, R.E., 2008, Space geodetic imaging of rapid ground subsidence in Mexico City: Geological Society of America Bulletin, 120, 1556-1566.

Cabral-Cano, E., Osmanoglu, B., Dixon, T., Wdowinski, S., DeMets, C., Cigna, F., Díaz-Molina, O., 2010, Subsidence and fault hazard maps using PS and permanent GPS networks in central Mexico:International Association of Hydrological Sciences, Publication Series, 339, 255-259.

Carreón-Freyre, D., Hidalgo-Moreno, C., Hernández-Marín, M., 2006, Mecanismos de fracturamiento de depósitos arcillosos en zonas urbanas: Caso de deformación diferencial en Chalco, Estado de México: Boletín de la Sociedad Geológica Mexicana, 58 (2), 237-250.

Carrillo, N., 1948, Influence of artesian wells in the sinking of Mexico City, en Procedures of the $2^{\text {nd }}$ International Conference on Soils Mechanics: Rotterdam, Holland, International Society for Soil Mechanics and Geotechnical Engineering, 2, 156-159.

Comisión Hidrológica de la Cuenca del Valle de México (CHVM), 1963, Estudios especiales, Secretaría de Recursos Hidráulicos: México D.F., reporte técnico, $84 \mathrm{p}$.

CONACyT-ICyTDF, 2012, Nivel de riesgo en la Ciudad de México en función de las características hidrogeológicas de la ZMCM y de fuentes potenciales y activas de contaminación por hidrocarburos. Proyecto FOMIX-CONACyT-GDF, clave 121128: Grupo de Hidrogeología, Facultad de Ingeniería, UNAM, reporte técnico, $176 \mathrm{p}$.

Ferretti, A., Prati, C., Rocca, F., 2001, Permanent scatterers in SAR interferometry: Geoscience and Remote Sensing, IEEE Transactions, 39, 8-20.

Ferretti, A., Fumagalli, A., Novali, F., Prati, C., Rocca, F., Rucci, A., 2011, A New Algorithm for Processing Interferometric Data-Stacks: SqueeSAR: Geoscience and Remote Sensing, IEEE Transactions, 49, 3460-3470.

Figueroa-Vega, G., 1984, Case history No. 9.8, Mexico, D.F., Mexico: Guidebook to studies of land subsidence due to ground-water withdrawal: Studies and Reports in Hydrology, 40, 217-232.

Galloway, D.L., Burbey, T.J., 2011, Review: regional land subsidence accompanying groundwater extraction: Hydrogeology Journal, 19, 1459-1486.

Galloway, D.L., Hoffmann, J., 2007, The application of satellite differential SAR interferometry-derived ground displacements in hydrogeology: Hydrogeology Journal, 15, 133-154.

Hernández-Espriú, A., Reyna-Gutiérrez, J.A., Sánchez-León, E., CabralCano, E., Carrera-Hernández, J., Martínez-Santos, P., MacíasMedrano, S., Falorni, G., Colombo, D, 2014, The DRASTIC-Sg model: an extension to the DRASTIC approach for mapping groundwater vulnerability in aquifers subject to differential land subsidence, with application to Mexico City: Hydrogeology Journal, $22,1469-1485$.

Herrera, I., Martínez, R., Hernández, G., 1989, Contribucion para la administracion científica del agua subterránea de la cuenca de Mexico: Geofísica Internacional, 28, 2, 297-334. 
Instituto Nacional de Estadística, Geografía e Informática (INEGI), 2011, Conteos de población y vivienda 2010: México, INEGI, revisado en julio del 2012, disponible en <http://www.inegi.org.mx/est/ contenidos/proyectos/ccpv/cpv2010/default.aspx>, consultado en mayo de 2013.

Lesser-Illades, J.M., González-Posadas, D., 2005, El Agua subterránea de la Ciudad de México, en V Congreso de Aguas Subterráneas (resumen): Hermosillo, Sonora, Asociación Geohidrológica Mexicana, 1-8.

Mazari, M., Ramírez, C., Alberro, J., 1992, Efectos de la extracción de agua en la zona de lacustre del valle de México: México D.F., Sociedad Mexicana de Mecánica de Suelos, AC., Raul J. Marsal, 37-48.

Mooser, F., 1975, Historia geológica de la Cuenca de México: Memoria de las Obras del Sistema de Drenaje Profundo del Distrito Federal: Mexico, D.F., Departamento del Distrito Federal, 1, 7-38.

Morales-Casique, E., Escolero, O. A., Arce, J.L., 2014, Resultados del pozo San Lorenzo Tezonco y sus implicaciones en el entendimiento de la hidrogeología regional de la cuenca de México: Revista Mexicana de Ciencias Geológicas, 31, 64-75.

Ortega-Guerrero, A., Cherry, J.A., Rudolph, D.L., 1993, Large-Scale Aquitard Consolidation Near Mexico City: Groundwater, 31, 708-718.

Ortega-Guerrero, A., Rudolph, D.L., Cherry, J.A., 1999, Analysis of longterm land subsidence near Mexico City: Field investigations and predictive modeling: Water Resources Research, 35 (11), 3327-3341.

Ortiz-Zamora, D., Ortega-Guerrero, A., 2010, Evolution of long-term land subsidence near Mexico City: Review, field investigations, and predictive simulations: Water Resources Research, 46, 1-15.

Santoyo, E., Ovando, E., Mooser, F., León, E., 2005, Síntesis geotécnica de la Cuenca del Valle de México: México D.F., TGC Ediciones, 171 p.

Sistema de Aguas de la Ciudad de México (SACM), 2006, Modelo hidrodinámico del acuífero de la Zona Metropolitana de la Ciudad de México: México, Instituto Mexicano de Tecnología del Agua (IMTA), Reporte Técnico 06-CD-03-1O-0272-1-06, 285 p.
Solano-Rojas, D., Cabra-Cano, E., Hernández-Espriú, J., Cigna, F., Wdowinsky, S., Osmanoglu, B., Falorni, G., Bohane, A., Colombo, D., 2012, Mapas de Riesgo por Fallamiento Asociado a subsidencia en las ciudades de México y Morelia (Resumen): Puerto Vallarta, Jalisco, Unión Geofísica Mexicana, Reunión Anual Unión Geofísica Mexicana, 292.

Tomás, R., Romero, R., Mulas, J., Marturià, J.J., Mallorquí, J.J., LopezSanchez, J.M., Herrera, G., Gutiérrez, F., González, P.J., Fernández, J., Duque, S., Concha-Dimas, A., Cocksley, G., Castañeda, C., Carrasco, D., Blanco, P., 2014, Radar interferometry techniques for the study of ground subsidence phenomena: a review of practical issues through cases in Spain: Environmental Earth Sciences, 71 (1), 163-181.

Tele-Rilevamento Europa (TRE), 2011, SqueeSAR: Milán, TeleRilevamento, actualizado en 2013, disponible en $<\mathrm{http}$ ://treuropa. com/technique/squeesar>, consultado en marzo de 2013.

Universidad Autónoma Metropolitana, 2005, Expansión histórica del Área urbana de la Ciudad de México: Observatorio Urbano de la Ciudad de México: México, UAM, actualizado en 2005, disponible en $<$ http://www.ocim.azc.uam.mx/EXPAN\%20MET/mwf/EXPAN\%20 ZMVM.mwf $>$, consultado en mayo de 2013.

Vázquez-Sánchez, E., Jaimes-Palomera, R., 1989, Geología de la Cuenca de México: Geofísica Internacional, 28, 133-190.

Zumberge, J., Heflin, M., Jefferson, D., Watkins, M., Webb, F., 1997, Precise point positioning for the efficient and robust analysis of GPS data from large networks: Journal of Geophysical Research: Solid Earth (1978-2012), 102, 5005-5017.

Manuscrito recibido: Mayo 12, 2014.

Manuscrito corregido recibido: Diciembre 1, 2014.

Manuscrito aceptado: Diciembre 5, 2014. 